\title{
Spatio-Temporal Semantic Representation of Cardiac MRI in Heart Attack Patients
}

\author{
D.N.F. Awang Iskandar and Hamimah Ujir \\ Faculty of Computer Science and Information Technology, \\ Universiti Malaysia Sarawak, \\ 94300 Kota Samarahan, Sarawak, Malaysia. \\ \{dnfaiz, uhamimah@fit.unimas.my\}
}

\begin{abstract}
Semantic Web technologies, applications and tools have made great steps forward in the life science and health care data exchange. However, developing appropriate semantic representations, including designing spatio-temporal ontologies, remains difficult and challenging. In this paper, we describe a framework to engineer a spatio-temporal semantic representation for the Cardiac MRI images using the current existing case studies conducted in Sarawak General Hospital Heart Centre.
\end{abstract}

Keywords- Cardiac MRI, Spatio-temporal representation, ontology

\section{INTRODUCTION}

Cardiac MRI images data generated by various hospitals and clinics worldwide is increasing at a steady rate and provides an opportunity for new computer science and information technology researches. Hospital and clinic administrators have developed awareness to the need of adequate cataloguing, representation and indexing the rapidly increasing MRI image databank.

Further supporting the awareness are researches that involves big data where data not only carries volume but also value and transforming data into knowledge. Cardiac MRI image data are collected using MRI machines for both medical and research usage. Transforming the data into knowledge requires involvement of computer science and information technology techniques to aggregate the data in hierarchically organised knowledge that will be more understood by non-experts, such as medical students and researchers from other domains.

In this paper we present a theoretical framework to represent the Cardiac MRI image databank in an ontology which will extend existing medical ontologies. Our main goal is to explore and study the techniques on how to bridge the spatio-temporal semantic gap problem in biomedical atlases using semantic web technology. We hope to achieve this goal by developing an ontology that can help to compute the correlation between the biomarkers and a series of cardiac MRI images of heart attack patients in determining the amount of scar healing and LV remodelling.
Biomedical atlases typically consist of a three main components. These components are the graphical model, the ontology that is associated with the graphical model and a mapping between those two. In this research, the graphical model that will be used is the Cardiac MRI images. An ontology is composed of objects and conceptual relations between the objects. Ontology is the theory of objects in terms of the criteria which allow one to distinguish between different types of objects and their relationships, dependencies, and properties. The spatiotemporal ontology contains spatial information of the cardiac physical and anatomical relations; and the temporal information is the sequence of clinical events and episodes of care. The mapping between the Cardiac MRI images and ontology would guide medical decision making as it can be used to characterise the state and behaviour of a patient's disease both in terms of natural course and as the result of therapeutic interventions. Hence, it also contributes as phenomenon-centric data to support health care research. We focus on Semantic Web technologies for spatio-temporal representation of the Cardiac MRI images alongside clinical data obtained from Sarawak General Hospital, Sunnybrook Cardiac MR Database [1], DETERMINE and MESA [2]. The objectives are to explore and study how to design, represent and engineer a spatio-temporal ontology for Cardiac MRI Images. Expected outcomes are the spatiotemporal semantic representation of the knowledge implicit in biomedical image data represented using an ontology. The ontology will be in RDF/OWL. The research will include prototyping of SPARQL end points to test querying the underlying data using the representations developed in this work.

The remainder of this paper is organised as follows. In Section II, we present the background on existing work. In Section III, we describe the case studies and data. In Section IV, we present and discuss the proposed framework, and conclude in Section $\mathrm{V}$ with a discussion of our suggestions for future work.

\section{RESEARCH BACKGROUND}

Research to detect, recognise and interpret Cardiac MRI images has started since the 1980's. Florentine et al. [3] noted that MRI is a non-invasive technique and does not require the use of ionising radiations. The images are tomographic, of 